\title{
Evolving bacterial envelopes and plasticity of TLR2-dependent responses: basic research and translational opportunities
}

\author{
Junbin $\mathrm{Li}^{\dagger}$, Dennis Sang Won Lee ${ }^{\dagger}$ and Joaquín Madrenas* \\ Microbiome and Disease Tolerance Centre, Department of Microbiology and Immunology, McGill University, Montreal, QC, Canada
}

\section{Edited by:}

Theodoros Kelesidis, University of

California Los Angeles, USA

\section{Reviewed by:}

Ranjit Kumar, University of Alabama at Birmingham, USA

Monica E. Embers, Tulane University, USA

Olivier Dussurget, University Paris Diderot, France

\section{*Correspondence:}

Joaquín Madrenas, Microbiome and

Disease Tolerance Centre,

Department of Microbiology and

Immunology, McGill University, 3775

University Street, QC H3A 2B4,

Canada

e-mail: joaquin.madrenas@mcgill.ca

${ }^{\dagger}$ Junbin Li and Dennis Sang Won Lee have contributed equally to this work.
Innate immune mechanisms that follow early recognition of microbes influence the nature and magnitude of subsequent adaptive immune responses. Early detection of microbes depends on pattern recognition receptors that sense pathogen-associated molecular patterns or microbial-associated molecular patterns (PAMPS or MAMPs, respectively). The bacterial envelope contains MAMPs that include membrane proteins, lipopeptides, glycopolymers, and other pro-inflammatory molecules. Bacteria are selected by environmental pressures resulting in quantitative or qualitative changes in their envelope structures that often promote evasion of host immune responses and therefore, infection. However, recent studies have shown that slight, adaptive changes in MAMPs on the bacterial cell wall may result in their ability to induce the secretion not only of pro-inflammatory cytokines but also of anti-inflammatory cytokines. This effect can fine-tune the subsequent response to microbes expressing these MAMPs and lead to the establishment of a commensal state within the host rather than infectious disease. In this review, we will examine the plasticity of Toll-like receptor (TLR) 2 signaling as evidence of evolving MAMPs, using the bettercharacterized TLR4 as a template. We will review the role of differential dimerization of TLR2 and the arrangement of signaling complexes and co-receptors in determining the capacity of the host to recognize an array of TLR2 ligands and generate different immune responses to these ligands. Last, we will assess briefly how this plasticity may expand the array of interactions between microbes and immune systems beyond the traditional disease-causing paradigm.

Keywords: toll-like receptors, gram-positive bacteria, commensal bacteria, microbiota, immune response

\section{INTRODUCTION}

Innate immunity is characterized by an immediate response against pathogens and is paramount in the initial control of infection. Toll-like receptors (TLRs) are thought to be among the most ancient pathogen recognition systems (1). Such systems recognize pathogen- or microbial-associated molecular patterns (PAMPs or MAMPs) that are relatively conserved among microorganisms but are absent in hosts. This recognition involves pattern recognition receptors (PRRs) that are broadly expressed by a variety of immune cells such as monocytes, macrophages, dendritic cells (DCs), and some epithelial cells. PRR-dependent responses are critical for promoting inflammatory processes leading to microbial clearance.

The genes encoding receptors of the innate immune system are inherited and not subject to gene rearrangement like those coding for adaptive immune receptors. As a result, the innate response has been traditionally considered fairly static in its recognition of microbial patterns, and to be largely indiscriminate in promoting inflammation and priming adaptive immunity. However, emerging evidence indicates that PRR signaling can also promote anti-inflammatory responses under certain conditions (2-5). The precise role of PRR-induced anti-inflammatory responses in the context of innate immunity remains uncertain.
Recent evidence suggests that these anti-inflammatory responses may play an important role in the regulation of the homeostatic tolerance to commensal organisms or uncontrolled microbial proliferation (6).

Two views of the biology of PRR-induced responses may be considered. One is that host responses to MAMPs vary depending on the context of exposure (e.g., site, responding cell type, expression of co-receptors by responding cell, etc.), which ultimately determines the type of response (pro- vs. anti-inflammatory responses). Alternatively, we should not forget that MAMPs are subject to evolutionary modification as a result of selective pressures by host defense mechanisms, and that this evolution will ultimately expand the array of ligands to a given PRR and the signals that it can deliver. However, co-evolution of both MAMPs as well as host responses can explain the two opposite outcomes of PRR signaling. In this review, we will focus only on TLRs, and specifically on TLR2 and TLR4, to review the plasticity of signaling by these receptors and its biological implications.

\section{OVERVIEW OF TOLL-LIKE RECEPTORS}

Toll-like receptors were the first class of PRRs identified. In 1989, Charles Janeway hypothesized that the innate immune 
system senses the presence of microbes by recognizing commonly associated molecular signatures (7). In 1996, his hypothesis was vindicated with the discovery of the Toll pathway in Drosophila and its role in controlling fungal infections (8) and subsequently with the characterization of associated PRRs Gram-negative bacteria binding protein 3 (GNBP3) and peptidoglycan (PGN) recognition protein SA (PGRP-SA), which recognize bacterial and fungal MAMPs (9, 10). Soon after, Medzhitov et al. (11) discovered a human homolog for Toll, at the time termed hToll, which was found to activate the transcription factor NF- $\mathrm{B}$. Mice deficient in this receptor were unable to induce pro-inflammatory cytokines to lipopolysaccharide (LPS) (12). This receptor, later called TLR4, was the first member of the TLR family of receptors to be characterized, a family that now includes 13 members, 10 of which are expressed in humans (13).

Structural studies have characterized TLRs as type I transmembrane proteins with leucine-rich repeats (LRRs) on the extracellular N-terminal domain (13). This LRR domain contains an $\alpha$-helix and a $\beta$-strand linked by loops, leading to the prediction that the ectodomain of TLRs assume a horseshoe conformation (14). The intracellular C-terminal portion of TLRs contains a Toll/interleukin-1 receptor (TIR) domain, which is common to all members of the TLR family (15).

Toll-like receptors are constitutively expressed on monocytes, macrophages, and DCs, while certain TLRs are also expressed on other cell types such as neutrophils, mast cells, epithelial cells, and B cells (13). TLR1, 2, 4, 5, 6, and 10 are expressed on the cell surface, while TLR 3, 7, 8, and 9 are expressed in intracellular compartments such as the endosome, lysosome, and the endoplasmic reticulum (1). TLRs 1-9 are conserved between humans and mice. TLR10 is a non-functional pseudogene in mice due to a retroviral insertion, but is nonetheless a functional receptor in humans (16). TLRs 11,12 , and 13 are poorly characterized and are absent from the human genome (17).

Through TLRs, the host can recognize a wide variety of microbial ligands including nucleic acids, lipids, lipoproteins, and polysaccharides. TLRs can be grouped according to their recognition of similar MAMPs. For example, TLRs 3, 7, 8, 9, and 13 all recognize nucleic acids. However, TLRs can also recognize a repertoire of structurally unrelated ligands. TLR4 has long been recognized as the receptor for LPS, but also recognizes heat-shock proteins (18), glycoproteins such as fibronectin (19), the fusion protein of respiratory syncytial virus (RSV) (20), and the plant diterpene and chemotherapy drug paclitaxel $(21,22)$. Similarly, TLR2 can recognize lipoteichoic acid (LTA), PGN, lipopeptides, and zymosan (reviewed in Ref. (13) and see below). Unlike TLR4 which signals as a homodimer, TLR2 forms heterodimers with TLR1, TLR6, and perhaps TLR10, hinting at a potential mechanism to discriminate microbial ligands and elicit varied downstream responses.

Toll-like receptors function as important immune receptors that, in coordination with other PRRs, turn on innate mechanisms of immunity, including inflammation. It is thus expected that changes in TLR ligand binding and signaling capacity will translate in changes in innate immunity. This is well illustrated by observations that certain polymorphisms in TLRs have been associated with increased susceptibility to a myriad of infectious diseases (23, 24) and to some non-infectious diseases such as cancer (25-27).

\section{MOLECULAR BASIS OF TLR2 AND TLR4 SIGNALING}

Since the discovery of TLRs, signaling from these receptors has been recognized as potently eliciting pro-inflammatory responses in the host (15). The bacterial endotoxin LPS found in the Gramnegative bacterial cell wall, and lipopeptides and glycopolymers in the PGN layer of Gram-positive bacterial cell wall are potent stimulants of inflammation, following TLR4 and TLR2 recognition respectively. PGN can stimulate inflammation via other PRRs such as nucleotide-binding oligomerization domain-containing protein (NOD1) and NOD2. Inflammation is characterized by the production of a vast array of cytokines including IL-1 $\beta$, IL-6, and $\mathrm{TNF} \alpha$, as well as chemokines such as IL-8 and MCP-1/CCL-2, and type I interferons (IFN) through the NF- $\kappa$ B and IRF3 transcription factors. The observation that down-regulation of TLR2mediated signaling with transmembrane domain-derived peptides reduces lethality in mice following intraperitoneal challenge with Staphylococcus aureus (28) illustrates that these pro-inflammatory mediators may be deleterious to the host when left unchecked.

Signaling from TLR2 and TLR4 is initiated by their ligandinduced dimerization. This step brings the TIR domains in their cytoplasmic tails into close proximity, forming a platform for signaling through TIR domain-containing adaptor molecules. TLR2 and TLR4 are phosphorylated on tyrosine residues, and deficiencies in their phosphorylation are associated with defective dimerization and impaired recruitment of TIR domain-containing adaptors (29, 30). Five TIR domain-containing adaptors have been identified: MyD88, MAL/TIRAP, TRIF, TRAM, and SARM [reviewed in Ref. (31)]. MyD88 was the first such adaptor discovered, and is involved in the signaling of all the TLRs except TLR3. TLR4 is known to utilize two distinct signaling pathways (MyD88-dependent and MyD88-independent pathways), mediated by different TIR domain-containing adaptor molecules, and leading to the induction of pro-inflammatory cytokines through mitogen-activated protein (MAP) kinase and NF- $\mathrm{B}$ activation. However, MyD88-deficient mice challenged with LPS were still able to induce the activation of NF- $\mathrm{B}$ and JNK (a MAP kinase) with a delayed response (32). This MyD88-independent, TRIFdependent pathway was later shown to induce pro-inflammatory cytokines as well as type I IFN through IRF3 (33). TRIF was found to interact with both TLR3 and TLR4, but TRIF-deficient mice did not produce type I IFN in response to TLR3 or TLR4 ligands $(34,35)$. In contrast to TLR4, TLR2 mostly depends on the adaptors MyD88 and TIRAP/MAL for signaling $(36,37)$. Mice deficient in MAL displayed a similar phenotype to MyD88 knockout mice when challenged with LPS, exhibiting delayed activation of NF- $\mathrm{B}$ and MAPKs (37). MAL is thought to be particularly important for TLR2 signaling, as activation of NF- $\mathrm{B}$ and MAPKs is impaired to a greater extent in MAL-deficient cells following PGN stimulation compared to LPS stimulation (36). Additional roles for cell-type-specific TLR2 adaptors have been reported. For example, peritoneal but not bone-marrow-derived macrophages can produce IFN- $\beta$ through IRF3 and IRF7 in manner independent of MAL but dependent on TLR2 and TRIF (38). 


\section{DIFFERENTIAL SIGNALING BY GRAM-NEGATIVE BACTERIA: AN INDICATION FOR EVOLVING INNATE IMMUNE RECOGNITION}

Lipopolysaccharide is the major component of the outer leaflet of the Gram-negative cell envelope $(12,39,40)$ and as such is the most abundant Gram-negative MAMP capable of inducing an immune response. LPS is composed of an $\mathrm{O}$-antigen polysaccharide chain, an oligosaccharide core, and a lipid A anchor (41). The O-antigen polysaccharide projects away from the bacterial cell and its structure is highly variable from strain to strain. The oligosaccharide core component serves as a linker between the $\mathrm{O}$-antigen and the lipid A anchor, which is the primary mediator of LPS toxicity through TLR4-mediated recognition and signaling, causing fever, diarrhea, and septic shock (42).

Lipid A, however, is not absolutely conserved across species. Hexa-acylated forms of lipid A found in Escherichia coli and Salmonella enterica serovar Typhimurium are potent activators of TLR4 $(43,44)$. This pro-inflammatory response is demonstrated by the production of IL- $1 \beta$, IL- 6 , IL-12p 40 , and TNF $\alpha$ by monocytes and DCs leading to the organization of an inflammatory response at the site of infection (42). Conversely, tetra-acylated forms found in Helicobacter pylori and some forms of Pseudomonas aeruginosa do not activate TLR4 to a similar extent (45-47). Additionally, LPS species from Porphyromonas gingivalis induce TNF $\alpha$ and IL- 1 but not IL-12 and IFN- $\gamma$ via a TLR2-mediated mechanism, and LPS from Rhodobacter sphaeroides does not induce proinflammatory cytokine production (48). These species- and/or strain-specific differences point to diversity in TLR4 ligands as well as the versatility of innate immune receptors in cross-recognition of diverse MAMPs. Such diversity and versatility is consistent with the evolving nature of the bacterial cell wall.

In addition to differences in stimulatory capacity of LPS between bacterial strains, some microbes alter their lipid A moieties in response to environmental cues, a change that also translates into diversity in host recognition and response (49). This phenomenon is well documented for the PhoP/PhoQ two-component system in Salmonella spp. (50). PhoQ, located on the inner membrane, is activated by anti-microbial peptides, low magnesium concentrations, and/or acidic $\mathrm{pH}$ (51). Following activation, PhoQ phosphorylates and activates the transcriptional regulator PhoP. One of PhoP's effector functions results in the modification of lipid A by the 3-O-deacylase, PagL, and a palmitoyl transferase, PagP. PagL modifies lipid A by removing one fatty acid chain and creating a penta-acylated lipid A $(52,53)$. In contrast, PagP modifies lipid A by adding an additional fatty acid chain, creating a hepta-acylated lipid A. When both enzymes modify a lipid A moiety, the end result is a hexa-acylated lipid A with one less acyl chain linked to the core region and an additional acyl chain linked via another fatty acid. Kawasaki et al. (53) identified that the lipid A products of PagL and/or PagP led to a decrease in TLR4-mediated NF- $\kappa \mathrm{B}$ activation. This PhoP/PhoQ two-component system identifies just one example of a modification in S. enterica lipid A that modulates the innate immune response. This system is thus a good example to illustrate the evolving nature of the bacterial cell wall.

Lipopolysaccharide has also been reported to induce the production of the anti-inflammatory cytokine IL-10 in co-cultures of T cells with stimulated DCs as well as in primed DCs (54).
For example, LPS from Pseudoalteromonas strains was able to modulate the pro-inflammatory response to E. coli LPS owing to a combination of low immunostimulatory activity as well as competition for TLR4 (55). Co-stimulation with the two types of LPS resulted in lower pro-inflammatory cytokine production and higher IL-10. This indicated an additional potential role for LPS from commensal bacteria in maintaining immune homeostasis and preventing inflammatory diseases.

The evidence discussed above suggests that a single MAMP from different species can take on various forms and possess different immunostimulatory capacity via the same PRR based on modifications induced by environmental stimuli. Furthermore, a potential regulatory role may be played by LPS with regards to competition for TLR4 and induction of IL-10 in a mechanism that has yet to be elucidated. Experimental models of inflammatory bowel disease (IBD) have helped to identify genetic factors that reveal host susceptibilities to impairment of gut immune homeostasis (38). However, the products and components of the microbiota, such as LPS, may play a larger role in setting the balance of organisms in a given microbiota and the maintenance of the local immune environment $(6,56)$.

\section{ANTI-INFLAMMATORY TLR4 SIGNALING}

Although the pro-inflammatory effects of TLR4 signaling have been described in detail, the anti-inflammatory responses induced by TLR 4 activation are much less characterized. The cell compartment where TLR4 signaling occurs seems to be an important factor when it comes to differentiating between pro- and anti-inflammatory signaling. Contrary to TLR4 pro-inflammatory signaling at the cell surface, TLR4 signaling from endosomal compartments induces the secretion of the anti-inflammatory cytokine IL-10 (2). This compartment-dependent effect is due to a shift from MyD88/MAL-dependent pro-inflammatory signaling at the cell surface to TRIF/TRAM-mediated anti-inflammatory signaling in the endosome, and is mediated by the p110 $\delta$ isoform of PI3-kinase (PI3K). This PI3K isoform is involved in the TLR4 internalization, dissociation of MAL from the cell membrane, and subsequent degradation of MAL by calpain (2). Therefore, a shift in adaptor molecule use is associated with a shift in cytokine expression (Figure 1): the MyD88-dependent pathway leads to the production of inflammatory cytokines such as IL-1 $\beta$, IL- 6 , and TNF $\alpha$, whereas the TRIF-dependent pathway induces IFN- $\beta$ and IL-10. This model is corroborated by the observation that inactivation of p110 $\delta$ results in higher levels of the pro-inflammatory cytokines and lower levels of IFN- $\beta$ and IL-10.

Other signaling events downstream of TLR4 activation leading to this anti-inflammatory response are unclear. The MAP kinases p38 and JNK have been shown to be necessary for IL-10 secretion by bladder epithelial cells (BECs) (57). Inhibition of p38 and JNK in LPS-activated BECs reduces STAT3 expression, and this enhances IL-10 secretion (58). Therefore, the balance between pro- and anti-inflammatory TLR4 signaling may depend not only on the use of either the MyD88/MAL or TRIF/TRAM adaptor proteins respectively, but also on additional factors. TRIF also plays a role in pro-inflammatory signaling, as evidenced by the complete abrogation of LPS-stimulated NF- $\kappa \mathrm{B}$ and JNK activation in MyD88 and TRIF double knockout mice (35) but not in 


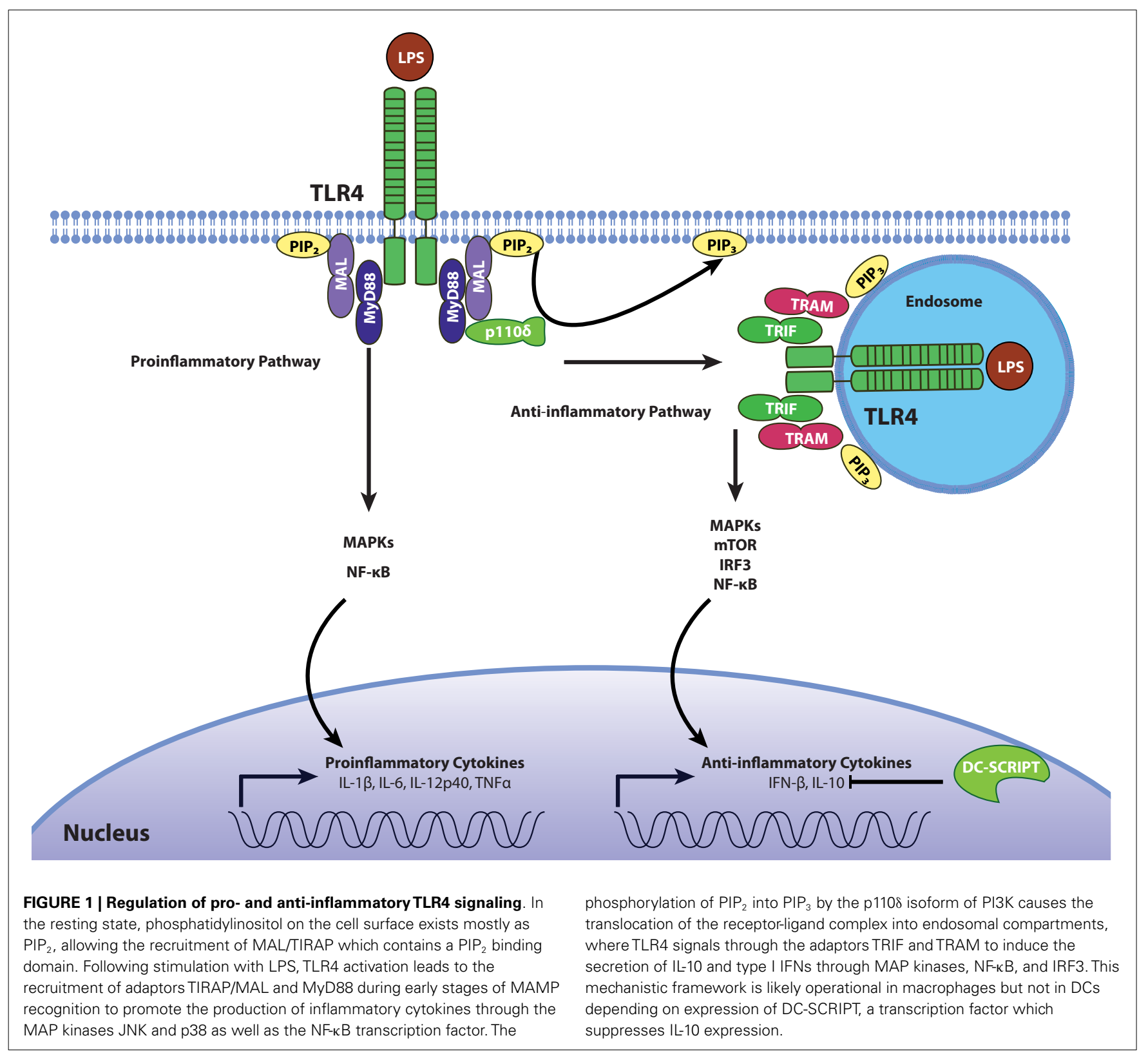

mice deficient for MyD88 alone, although the latter have delayed kinetics compared to wild-type control mice (32). Furthermore, quantitative proteomic analysis of the secretome of LPS-activated murine bone-marrow-derived macrophages suggests that both MyD88 and TRIF are required for full IL-10 expression, since IL-10 secretion was at least 100-fold greater when both adaptors were present compared to conditions in which only one was present (59).

\section{AN EXPANDING ARRAY OF TLR2 LIGANDS}

TLR2, in conjunction with other TLRs (see below), has been identified as a receptor for an array of ligands including PGN-embedded LTA, di- and tri-acylated lipopeptides, lipoproteins, and others including LPS variants $(60,61)$, zymosan from fungi, lipoarabinomannan from mycobacteria, glycosylphosphatidyl inositol mucin from Trypanosoma cruzi, hemagglutinin protein from measles virus, and phospholipomannan from Candida albicans [reviewed in Ref. (13)]. The envelope of Gram-positive bacteria contains a multitude of molecules that can act as MAMPs. These include the glycopolymers wall teichoic acid (WTA) and LTA, PGN fragments, lipopeptides and lipoproteins, and other proteins (40).

For years, LTA has been touted as an LPS equivalent for Grampositive bacteria by acting through a TLR2-mediated mechanism. LTA is a structural glycopolymer within the cell wall that is anchored to the cell membrane but protrudes outward through the cell wall (40). It is constituted of a polymer of repetitive 1,3-phosphodiester-linked glycerol-1-phosphate units with a glycolipid anchor. Some glycerol phosphate subunits are substituted with D-alanine residues, and are responsible for the stimulating properties of this molecule when tested with synthetic LTA (62). 
This substitution is also partially responsible for the resistance of some Gram-positive species to anti-microbial peptides (63). The glycolipid anchor often contains two acyl chains that may be responsible for binding to TLR2 (62). The precise contribution of LTA to the overall TLR2-triggered response is uncertain (64). It has been reported that only lipoproteins and lipopeptides can induce TLR2 signaling under physiological concentrations and that other cell wall fractions (such as the LTA fraction) are contaminated by these molecules $(64,65)$.

Although both lipoproteins and PGN are found in both Gramnegative and Gram-positive species, the magnitude of LPS-TLR4 interaction often overshadows the immunostimulatory capacity of lipoproteins in Gram-negative bacteria. Although lipoproteins are likely a primary bacterial ligand for TLR2 (64), most experimental data on TLR2 function has been generated using synthetic lipopeptides. Structural studies using the synthetic lipopeptides Pam2CSK4 and Pam3CSK4 show that TLR2 dimerized with TLR1 recognizes triacylated lipoproteins while TLR2-TLR6 dimers recognize diacylated lipoproteins $(66,67)$. This functional distinction aligns with the structural conformation observed from the crystal structures of these heterodimers (66). Intriguingly, the acyl chains of the lipoproteins, which are hypothesized to bind to TLRs, are embedded into the bacterial membrane, making it uncertain whether they are accessible to immune receptors. In Gram-positive bacteria, the lipoproteins are found below the PGN wall but on the outer leaflet of the cytoplasmic membrane (40). In Gram-negative bacteria, the lipoproteins are found on the outer leaflets of the inner and outer membranes. However, Boneca et al. (68) have shown that phagocytosis and proteolytic destruction of $S$. aureus by macrophages may be necessary for TLR-mediated responses and in this way contribute to the generation of ligands for TLR2 or NOD receptors that amplify the innate response to these bacteria. Furthermore, some triacylated lipoproteins have been found to induce an immune response dependent on TLR2 but independent of TLR1 or TLR6 (69). Of interest, environmental conditions may impact the balance between di- vs. tri-acylated lipopeptide expression by bacteria and, thus influence the type of ensuing responses (70). Gram-positive bacteria can also modify their MAMPs in response to selective pressures applied by the host immune system. Listeria monocytogenes peptidoglycan $N$ deacetylase $(\operatorname{PgdA})$ decreases its recognition by innate immune receptors, playing a role in immune evasion. Strains with this gene knocked out displayed increased sensitivity to lysozyme, impaired survival in macrophages, and impaired virulence in vivo in $\mathrm{BALB} / \mathrm{c}$ and C57/BL6J mice (68). Based on the heterogeneity of TLR2 ligands, it is plausible to suggest that there may be an array of ligands for TLR2 that results from environmental selection upon microbial-host interactions. If this is the case, then fine characterization of mechanisms involved in TLR2-mediated recognition and signaling may turn out to be a much more laborious exercise than initially thought.

\section{PLASTICITY IN TLR2 SIGNALING}

As mentioned above, the MAMPs that can act as TLR2 ligands (in association with TLR1, TLR6, and maybe TLR10) are many and structurally diverse, albeit sharing lipid moieties (13). The variation in structure and biological origins suggest that there is a considerable plasticity in TLR2-dependent recognition and signaling. Indeed, like TLR4, TLR2 has recently been recognized as capable of eliciting anti-inflammatory cytokine responses (3-5). For example, upon stimulation with staphylococcal PGN preparations, peripheral blood mononuclear cells (PBMCs) produced IL-10 through a TLR2-dependent mechanism, down-regulating the T cell response to staphylococcal superantigens (69). Further studies indicated that the PI3K-Akt pathway was indispensible for IL-10 production, as the IL-10 response was associated with Akt phosphorylation and was inhibited by the PI3K inhibitor wortmannin (71).

Unlike TLR4 which signals as a homodimer, TLR2-dependent MAMP recognition and signaling requires, under most circumstances, formation of TLR2 heterodimers. TLR2 is thought to exist in pre-formed low affinity complexes associated with TLR1 and TLR6 under basal conditions and dimerizes upon ligand binding (66), heterodimerizing with TLR1 or TLR6 upon recognition of triacylated and diacylated lipopeptides respectively $(72,73)$. Such a heterodimerization of TLR2 has been considered a factor potentially determining the ensuing pro- vs. anti-inflammatory responses. In general, TLR2/1 complexes have been more often linked with pro-inflammatory responses than TLR2/6 complexes, which have been linked with anti-inflammatory responses $(4,74)$. The structural basis for this difference is unknown at the moment. In addition, it has been claimed that TLR2 homodimers may down-regulate TLR2-dependent responses. However, the evidence for this claim is solely based on in vitro recombinant systems, using chimeric proteins of the extracellular domain of CD4 fused with the transmembrane and intracellular domains of TLR2, and may not take into account other regulatory factors associated with natural ligand-induced TLR2 dimerization (72).

Other cell surface PRRs that could associate with TLR2 can also contribute to the different functional outcomes of TLR2 engagement. SitC, a triacylated lipoprotein found in S. aureus, was recently shown to induce IL-6 and TNF $\alpha$ through TLR2 and MyD88 in peritoneal macrophages (75). This was observed even in mice deficient in TLR1 and TLR6, suggesting that TLR2 can signal through dimers other than TLR2/1 and TLR2/6, either TLR2 homodimers or, alternatively, TLR2 dimerizing with other PRRs. In human cells, one such candidate could be TLR10. Although not expressed in mouse cells, TLR10 is a functional MyD88-dependent receptor in humans, being expressed on B cells and certain DC subsets (16). In 2001, TLR10 was identified as a receptor with the characteristic LRRs and Toll/IL-1 receptor TIR domains shared by all TLRs (76). Importantly, this study found that TLR10 was highly homologous to TLR1 and TLR6 with an overall amino acid identity of 50 and $49 \%$ respectively, whereas it was only $30 \%$ identical to TLR2 and no more than $25 \%$ for the other TLRs. Phylogenetic studies on TLR evolution indicate that TLR10 predates TLR1 and TLR6, suggesting that TLR1 and TLR6 arose from gene duplication, consistent with the observation that TLR10, TLR1, and TLR6 lie in tandem on human chromosome 4 (77). It has been suggested that TLR10 shares ligand recognition with TLR1 but signals differently (78). Given that TLR10 polymorphisms have been associated with various human pathologies (79-81), TLR10 represents a promising direction in which to increase our understanding of the plasticity of TLR2 responses. 
Another factor to consider in the signaling properties of TLR2 is the availability of co-receptor molecules. Among these, CD14 (82) and CD36 (83) are accessory molecules known to contribute to certain responses to TLR2 signaling [reviewed in Ref. (84)]. Availability of these molecules is important for pro-inflammatory responses to TLR2 ligation but not for IL-10 production (71). These accessory proteins are known to bind to TLR ligands. CD14, for example, is able to bind LPS, PGN $(85,86)$, Pam3CSK4 (87), polyI:C (88), and CpG DNA (89). They also play a role in ligand discrimination, as loss of CD36 impairs the TNF $\alpha$ response against LTA and the TLR2/6 ligand MALP2, but not Pam2CSK4, Pam4CSK4, LPS, PGN, zymosan, polyI:C, or CpG DNA (90). MD2 has also been shown to associate with both TLR4 and TLR2 to enhance signaling by their cognate ligands (91). It is unknown whether binding of these accessory proteins changes the conformation of the intracellular domains of TLRs and, if so, how this affects signaling. The use of peptides mimicking the intracellular domains of TLRs offers a promising platform to study TLR dimerization and receptor assembly (28).

Although PI3K has been shown to regulate the balance of provs. anti-inflammatory responses following TLR4 activation, the precise regulatory role of $\mathrm{PI} 3 \mathrm{~K}$ in the regulation of pro- vs. antiinflammatory signaling from other TLRs, and specifically from TLR2, remains untested. While PI3K-dependent regulation of TLR4 anti-inflammatory signaling has been shown to involve differential compartmentalization of the TLR complex, TLR2 only induces TNF $\alpha$ and type I IFN from endosomal compartments (56, 92). Like TLR4, TLR2 also uses the MAL adaptor molecule, and activation of TLR2/ 6 with diacylated lipopeptides has been shown to lead to MAL-p85 $\alpha$ interaction and Akt-mediated macrophage polarization, suggesting a potential mechanism for TLR2 signaling plasticity (93). However, for TLR2, the regulatory role of PI3K may not involve differential compartmentalization of signaling complexes (Peres and Madrenas, unpublished observations).

Sustained ligation of TLR2 with TLR1, TLR6, or TLR10 brings the TIR domains within close proximity, forming the platform for TIR adaptors, and leads to downstream signaling events. Availability of intracellular adaptors is another factor that can accommodate different signaling patterns from TLR2. For antiinflammatory responses, we already mentioned that TLR2/ 6 heterodimers can use the MAL adaptor molecule leading to PI3K-Akt activation, which includes IL-10 production among its functional correlates (93). As previously mentioned, certain macrophage populations have been shown to induce IFN- $\beta$ through a TLR2 and TRIF-dependent mechanism (38). Type I IFN have been suggested as initiators of the IL-10 response, but it is not clear if there is a mechanistic link. However, PBMCs stimulated with heat killed $S$. aureus may not require type I IFN for the IL-10 response (Peres and Madrenas, unpublished observations). Another adapter linked to TLR-induced PI3K activation is the B-cell adapter for PI3K (BCAP). It has been shown that the TIR domain of BCAP interacts with MyD88 and MAL to suppress production of proinflammatory cytokines in bone-marrow-derived macrophages $(94,95)$ (Figure 2). Mice deficient in BCAP showed impaired Akt phosphorylation in response to stimulation with LPS, CpG, and Pam3CSK4 (94), but the phosphorylation of the MAP kinases extracellular-signal regulated kinase (ERK) and JNK in response to LPS was not affected (95).
Interestingly, $\mathrm{Ni}$ et al. (95) found that IL-10 production after LPS stimulation did not differ between the BCAP-deficient and control groups, seemingly contradicting the findings in $\mathrm{p} 110 \delta$ enzymatically deficient mice (2). Further investigation is required on the possible differential role of PI3K in TLR4- and TLR2mediated anti-inflammatory responses. Since PI3K constitutes a relatively large family of enzymes comprising three classes, each with several isoforms that serve diverse cellular functions, one cannot rule out that the plasticity in TLR signaling results, in part, from different usage of these isoforms. Use of chemical inhibition of the PI3K/Akt pathway has its limitations. For example, the often-used wortmannin is a broad PI3K inhibitor that may impact other cellular functions. Therefore, further investigation is required to determine the mechanistic role of different PI3K isoforms in the TLR2-dependent anti-inflammatory response.

Toll-like receptor 2 signaling plasticity can also result from the specific stage of differentiation of the cell expressing this receptor. The main cell candidate responsible for the IL-10 response in human PBMCs is the CD14 ${ }^{+}$monocyte. Experiments using in vitro differentiation of primary monocytes into macrophages and DCs indicate that macrophages, not DCs are the primary mediators of the immunomodulatory or anti-inflammatory response (71). Recently, a potential mechanism has been hypothesized that could explain this difference in TLR2-dependent IL-10 secretion. DC-SCRIPT is a transcription factor found in all DC subsets, including in vitro monocyte-derived DCs, myeloid DCs, plasmacytoid DCs, and Langerhans cells, but is not expressed in other leukocytes (96). When DC-SCRIPT was knocked down using siRNA, the IL-10 levels were increased compared to controls in response to stimulation with LPS and R848, a TLR7/8 ligand (97). In contrast, the IL-10 response to poly I:C was unchanged (98). Unfortunately, TLR2 ligands were not examined in this study. DC-SCRIPT is a negative regulator of IL-10 production following activation of certain TLRs and represents a plausible explanation for the differences observed between monocyte-derived macrophages and DCs. Further investigation is required to characterize anti-inflammatory TLR2 responses in both monocyte-derived DCs and in vivo DC populations.

Considering all the factors mentioned above, we propose that the plasticity of TLR2 signaling is a reflection of the evolving nature of the bacterial cell wall. As a result of the selective pressure exerted on the microbes by the interacting hosts, the array of ligands for TLR2 increases. The versatility accumulated by the immune system over the phylogeny of the species is thus revealed by the interaction with these ligands. The outcome of these interactions may involve and, indeed, favor the microbial capacity to induce anti-inflammatory responses not only as an immune evasion mechanism, which ultimately may eliminate the host and negatively impact the microbe, but also as a way to promote pathobiosis or even commensalism.

\section{THERAPEUTIC POTENTIAL OF TLR2 SIGNALING PLASTICITY}

The evolving nature of MAMPs upon host-imposed selective pressure offers opportunities to explore novel immune modulatory mechanisms with therapeutic potential. The importance of TLR-induced anti-inflammatory pathways has been corroborated in vivo. For example, chronic recurrent multifocal osteomyelitis (CRMO) is an autoimmune bone disorder, and patients were 


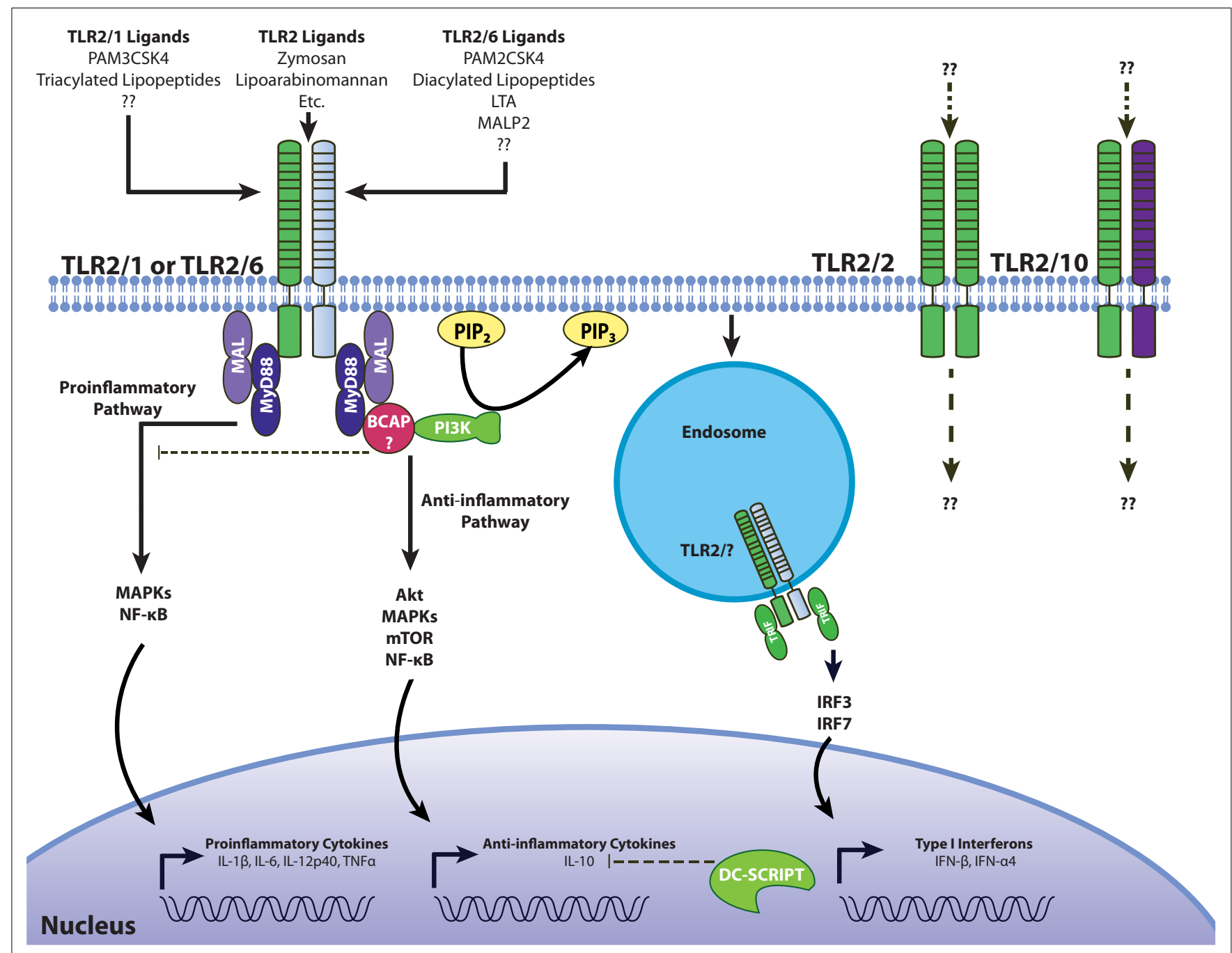

FIGURE 2 | Model for pro- and anti-inflammatory signaling through TLR2. Like TLR4, pro-inflammatory signaling through TLR2 involves the recruitment of the adaptors MyD88 and MAL/TIRAP to induce inflammatory cytokines through ERK, JNK, and NF-KB. BCAP interacts with both MyD88 and MAL to recruit $\mathrm{PI} 3 \mathrm{~K}$, which then phosphorylates $\mathrm{Akt}$, inducing the secretion of anti-inflammatory IL-10. The involvement of DC-SCRIPT in suppressing
TLR2-depdendent IL-10 production has not been tested. TLR2 activation can also induce type I interferons through IRF3 and IRF7 from endosomal compartments $(38,92)$. Differences in signaling between the TLR2/1 and TLR2/6 heterodimers, as well the potential involvement of hypothesized TLR2/2 and TLR2/10 receptor conformations presents another challenge in understanding the plasticity of TLR2-dependent responses. shown to have impaired IL-10 expression downstream of TLR4 (99). Hofmann et al. (99) showed that CRMO patients have deficient ERK 1 and 2 signaling following TLR4 activation. This results in an increase in the TNF $\alpha /$ IL-10 ratio, providing a possible mechanism for the pathogenesis of CRMO and suggests novel therapeutic targets. TLR2-dependent anti-inflammatory signaling may prove to be equally important in other clinical settings.

The lipopeptides that can act on TLR2 have garnered much attention as tools to understand the immune system and also as potential adjuvants because of the feasibility of their synthesis in the laboratory setting. Much of our understanding of TLR2 signaling comes from the use of the synthetic lipopeptides, Pam3CSK4, and Pam2CSK4, but it is unknown to what extent these ligands recapitulate the binding of and responses to physiological ligands. Pam3CSK4 and Pam2CSK4 were used to structurally characterize the binding of TLR2/1 and TLR2/6 to their cognate ligands $(66,67)$. Using $x$-ray crystallography, the binding profiles of human TLR2 were identified. TLR2 recognizes two acyl moieties on Pam2CSK4 or Pam3CSK4 while TLR1 recognizes one acyl moiety, and TLR6 does not have a binding pocket for any acyl chains. It is interesting to note that the accessory molecule CD14 has been reported to bind Pam3CSK4 and other triacylated lipopeptides and induce the formation of TLR2/1 signaling complexes (87). Presently, this finding has not been reported with Pam2CSK4. In addition, LPS binding protein (LBP) appears to play an independent and redundant role in Pam3CSK4 or bacterial lipoprotein presentation to the TLR2 signaling complex (100). Intriguingly, Pam2CSK4 signaled independently of TLR6 in murine bone marrow-derived macrophages and B lymphocytes (69). Currently, there is still no single defined natural ligand for 
TLR2. Furthermore, the differences in PRR signaling between mice and humans as well as ambiguities in potential ligands have yet to be elucidated. For now, it is believed that bacterial lipoproteins are the family of biomolecules responsible for TLR2 signaling (64).

Synthetic lipopeptides have also been examined as adjuvants (101). Pam3CSK4 showed promise for live-attenuated vaccines as it enhanced infection of paramyxoviruses in vitro and in vivo (102) as well as RSV infection in multiple cell types. However, the related lipopeptides Pam-Cys-SK4 and PHCSK4 did not activate TLR signaling although they were able to enhance the binding and infection of APCs by the virus, even though RSV primarily targets airway epithelial cells (103). The enhanced infectivity may allow live-attenuated vaccines to produce a more robust immune response and confer significantly stronger secondary responses $(102,103)$. In this regard, Pam-Cys-SK4 and PHCSK4 could be useful in the development of vaccines.

Synthetic lipopeptides targeting TLR2 have also been considered as potential modulators of $\mathrm{T}$ cell subset differentiation, which may be a strategy to confer protection to certain chronic inflammatory, immune mediated diseases or to enhance vaccine efficacy (104). For example, Pam3CSK4 enhanced CD8 ${ }^{+}$regulatory T cell $\left(\mathrm{T}_{\mathrm{reg}}\right)$ survival (105). Interestingly, glycolipopeptide constructs can be used to design composite tumor vaccines (106). These compounds contain carbohydrate antigens, $\mathrm{CD} 4^{+}$or $\mathrm{CD}^{+} \mathrm{T}$ cell epitopes, and TLR2 stimulating lipid chains. By compounding these properties, improved vaccines against malignant cancer cells may be generated. Also, by introducing structural modifications in native ligands it should be possible to enhance the anti-inflammatory properties of TLR2 signaling (107).

Finally, a role for TLRs (specifically TLR2) and other PRRs is emerging in the crosstalk between the host and its microbiome. TLR2 has been linked to the establishment and regulation of the microbiota in different sites. One such site is the skin, where staphylococcal LTA has been shown to down-regulate inflammatory cytokine release by keratinocytes in a TLR2-dependent manner (5). Another site is the gut, where microbiota has been shown to play an active role in the maturation and homeostasis of the host immune system $(108,109)$. Studies conducted on Bacteroides fragilis, a ubiquitous gut microbe, revealed that polysaccharide A (PSA) was involved in correcting CD4 ${ }^{+} \mathrm{T}$ cell deficiency and the $\mathrm{T}_{\mathrm{H}} 1 / \mathrm{T}_{\mathrm{H}} 2$ imbalance in germ-free mice (109). The significance of this mechanism has been demonstrated in disease models, where experimental colitis induced by Helicobacter hepaticus was more severe in $B$. fragilis $\triangle$ PSA compared to wild-type $B$. fragilis. Moreover, purified PSA protects animals from experimental colitis through an IL-10 dependent mechanism (110). PSA was found to signal through TLR2 directly on $\mathrm{T}_{\text {reg }}$ cells to promote immune

\section{REFERENCES}

1. Janeway CA, Murphy K, Paul T, Mark W. Janeway's Immunobiology. New York: Garland Science (2008).

2. Aksoy E, Taboubi S, Torres D, Delbauve S, Hachani A, Whitehead MA, et al. The pl10delta isoform of the kinase $\mathrm{PI}(3) \mathrm{K}$ controls the

tolerance by suppressing $\mathrm{T}_{\mathrm{H}} 17$ responses (6). It has been suggested that PSA is a member of a new class of TLR ligands known as symbiont-associated molecular patterns (SAMPs) where TLR signaling paradoxically may allow persistent colonization. These effects may not be limited to $B$. fragilis but be shared by other components of the microbiome (e.g., clostridia) $(111,112)$.

The immunological environment in the gut and other sites where the host interacts with its microbiome is a critical site for host-microbe co-evolution and dynamic selection of MAMPs and PRRs. There is an advantage for the host to evolve sophisticated mechanisms to differentially respond to molecular patterns associated with symbiosis or pathogenicity. The involvement of TLR2 in regulating the gut microbiota and preventing dysbiosis may be just one example of the complexity of TLR ligand co-evolution, providing additional impetus for further investigation of the plasticity of TLR2 signaling and potential avenues to exploit it.

\section{CONCLUSION}

While the pro-inflammatory responses activated upon detection of Gram-negative and Gram-positive bacteria by TLRs have been well characterized, the TLR-triggered anti-inflammatory responses are only beginning to be elucidated. The current literature regarding these new responses focus on TLR4 but TLR2 is especially interesting to study in this context given the diversity in receptor complexes and the crosstalk between downstream signaling cascades. The challenges ahead are not trivial: (i) the array of ligands for TLR2 may be larger than originally suspected, and its characterization may be an ongoing exercise as suggested by the evidence supporting their evolving nature; (ii) the relevance of various TLR2 ligands in the context of infection is not clear; and (iii) the tools used to study TLR2 activation both structurally and functionally are limited and may not recapitulate the full range of responses following TLR2 activation in vivo in response to bacteria. However, the therapeutic promise shown by synthetic TLR2 ligands as well as the emerging significance of TLR2 in regulating the gut microbiota, among other reasons, justify further investigation into the plasticity of TLR2-mediated MAMP recognition and downstream signaling.

\section{ACKNOWLEDGMENTS}

We thank the members of the Madrenas laboratory for helpful comments and criticisms on the manuscript. Also, we acknowledge our colleagues whose contributions to the field could not be included in the list of references due to space limitations. Research in the Madrenas laboratory is funded by the Canadian Institutes of Health Research. Joaquín Madrenas holds a Tier I Canada Research Chair in Human Immunology.

for interleukin 10-mediated immunosuppression. $J \quad \operatorname{Exp}$ Med (2002) 196:1017-24. doi:10.1084/jem.20020908

4. Chau TA, McCully ML, Brintnell W, An G, Kasper KJ, Vines $\mathrm{ED}$, et al. Toll-like receptor 2 ligands on the staphylococcal cell wall downregulate superantigeninduced $\mathrm{T}$ cell activation and prevent toxic shock syndrome. Nat Med (2009) 15:641-8. doi:10. 1038/nm.1965

5. Lai Y, Di Nardo A, Nakatsuji T, Leichtle A, Yang Y, Cogen AL, et al. Commensal bacteria regulate tolllike receptor 3-dependent inflammation after skin injury. Nat Med (2009) 15:1377-82. doi:10.1038/ nm.2062 
6. Round JL, Lee SM, Li J, Tran G, Jabri B, Chatila TA, et al. The toll-like receptor 2 pathway establishes colonization by a commensal of the human microbiota. Science (2011) 332:974-7. doi:10. 1126/science. 1206095

7. Janeway CA Jr. Approaching the asymptote? Evolution and revolution in immunology. Cold Spring Harb Symp Quant Biol (1989) 54(Pt 1):1-13. doi:10.1101/SQB. 1989.054.01.003

8. Lemaitre B, Nicolas E, Michaut L, Reichhart JM, Hoffmann JA. The dorsoventral regulatory gene cassette spätzle/toll/cactus controls the potent antifungal response in Drosophila adults. Cell (1996) 86:973-83. doi:10.1016/S00928674(00)80172-5

9. Kim YS, Ryu JH, Han SJ, Choi KH, Nam KB, Jang IH, et al. Gramnegative bacteria-binding protein, a pattern recognition receptor for lipopolysaccharide and beta-1,3glucan that mediates the signaling for the induction of innate immune genes in Drosophila melanogaster cells. J Biol Chem (2000) 275:32721-7. doi:10.1074/ jbc.M003934200

10. Werner T, Liu G, Kang D, Ekengren S, Steiner H, Hultmark D. A family of peptidoglycan recognition proteins in the fruit fly Drosophila melanogaster. Proc Natl Acad Sci U S A (2000) 97:13772-7. doi:10. 1073/pnas.97.25.13772

11. Medzhitov R, Preston-Hurlburt P, Janeway CA Jr. A human homologue of the Drosophila toll protein signals activation of adaptive immunity. Nature (1997) 388:394-7. doi:10.1038/41131

12. Poltorak A, He X, Smirnova I, Liu MY, Van Huffel C, Du X, et al. Defective LPS signaling in $\mathrm{C} 3 \mathrm{H} / \mathrm{HeJ}$ and $\mathrm{C} 57 \mathrm{BL} / 10 \mathrm{ScCr}$ mice: mutations in Tlr4 gene. Science (1998) 282:2085-8. doi:10. 1126/science. 282.5396 .2085

13. Akira S, Uematsu S, Takeuchi O. Pathogen recognition and innate immunity. Cell (2006) 124:783-801. doi:10.1016/j.cell.2006.02.015

14. Jin MS, Lee JO. Structures of the toll-like receptor family and its ligand complexes. Immunity (2008) 29:182-91. doi:10.1016/j.immuni. 2008.07.007

15. Bowie A, O'Neill LA. The interleukin-1 receptor/toll-like receptor superfamily: signal generators for pro-inflammatory interleukins and microbial products. $J$ Leukoc Biol (2000) 67:508-14.
16. Hasan U, Chaffois C, Gaillard C, Saulnier V, Merck E, Tancredi S, et al. Human TLR10 is a functional receptor, expressed by $\mathrm{B}$ cells and plasmacytoid dendritic cells, which activates gene transcription through MyD88. JImmunol (2005) 174:2942-50.

17. Kawai T, Akira S. The role of pattern-recognition receptors in innate immunity: update on tolllike receptors. Nat Immunol (2010) 11:373-84. doi:10.1038/ni.1863

18. Lee KH, Jeong J, Yoo CG. Positive feedback regulation of heat shock protein 70 (Hsp70) is mediated through toll-like receptor 4-PI3K/Akt-glycogen synthase kinase-3beta pathway. Exp Cell Res (2013) 319:88-95. doi:10.1016/j. yexcr.2012.09.018

19. Lefebvre JS, Levesque T, Picard S, Pare G, Gravel A, Flamand L, et al. Extra domain A of fibronectin primes leukotriene biosynthesis and stimulates neutrophil migration through activation of tolllike receptor 4. Arthritis Rheum (2011) 63:1527-33. doi:10.1002/ art.30308

20. Marr N, Turvey SE. Role of human TLR4 in respiratory syncytial virus-induced NF-kappaB activation, viral entry and replication. Innate Immun (2012) 18:856-65. doi:10.1177/1753425912444479

21. Kawasaki K, Akashi S, Shimazu R, Yoshida T, Miyake K, Nishijima M. Mouse toll-like receptor 4.MD-2 complex mediates lipopolysaccharide-mimetic signal transduction by Taxol. J Biol Chem (2000) 275:2251-4. doi:10.1074/ jbc.275.4.2251

22. Wang J, Kobayashi M, Han M, Choi S, Takano M, Hashino S, et al. MyD88 is involved in the signalling pathway for Taxolinduced apoptosis and TNF-alpha expression in human myelomonocytic cells. Br J Haematol (2002) 118:638-45. doi:10.1046/j.13652141.2002.03645.x

23. Schwartz DA, Cook DN. Polymorphisms of the toll-like receptors and human disease. Clin Infect Dis (2005) 41 (Suppl 7):S403-7. doi:10. 1086/431985

24. Misch EA, Hawn TR. Toll-like receptor polymorphisms and susceptibility to human disease. Clin Sci (Lond) (2008) 114:347-60. doi: 10.1042/CS20070214

25. El-Omar EM, Ng MT, Hold GL. Polymorphisms in toll-like receptor genes and risk of cancer. Oncogene (2008) 27:244-52. doi:10. 1038/sj.onc. 1210912
26. Kutikhin AG. Association of polymorphisms in TLR genes and in genes of the toll-like receptor signaling pathway with cancer risk. Hum Immunol (2011) 72:1095-116. doi:10.1016/j. humimm.2011.07.307

27. Roszak A, Lianeri M, Sowinska A, Jagodzinski PP. Involvement of toll-like receptor 9 polymorphism in cervical cancer development. Mol Biol Rep (2012) 39:8425-30. doi:10.1007/s11033-012-1695-8

28. Fink A, Reuven EM, Arnusch CJ, Shmuel-Galia L, Antonovsky N, Shai Y. Assembly of the TLR2/6 transmembrane domains is essential for activation and is a target for prevention of sepsis. J Immuno (2013) 190:6410-22. doi:10.4049/ jimmunol.1202033

29. Xiong Y, Song C, Snyder GA, Sundberg EJ, Medvedev AE. R753Q polymorphism inhibits toll-like receptor (TLR) 2 tyrosine phosphorylation, dimerization with TLR6, and recruitment of myeloid differentiation primary response protein 88 . J Biol Chem (2012) 287:38327-37. doi:10.1074/jbc.M112.375493

30. Medvedev AE, Piao W, Shoenfelt J, Rhee SH, Chen H, Basu S, et al. Role of TLR4 tyrosine phosphorylation in signal transduction and endotoxin tolerance. $J$ Biol Chem (2007) 282:16042-53. doi: 10.1074/jbc.M606781200

31. O'Neill LA, Bowie AG. The family of five: TIR-domain-containing adaptors in toll-like receptor signalling. Nat Rev Immunol (2007) 7:353-64. doi:10.1038/nri2079

32. Kawai T, Adachi O, Ogawa T, Takeda K, Akira S. Unresponsiveness of MyD88-deficient mice to endotoxin. Immunity (1999) 11:115-22. doi:10.1016/S10747613(00)80086-2

33. Barbalat R, Lau L, Locksley RM, Barton GM. Toll-like receptor 2 on inflammatory monocytes induces type I interferon in response to viral but not bacterial ligands. Nat Immunol (2009) 10:1200-7. doi: 10.1038/ni.1792

34. Yamamoto $M$, Sato S, Mori $\mathrm{K}$, Hoshino $\mathrm{K}$, Takeuchi $\mathrm{O}$, Takeda $\mathrm{K}$, et al. Cutting edge: a novel toll/IL-1 receptor domaincontaining adapter that preferentially activates the IFN-beta promoter in the toll-like receptor signaling. J Immunol (2002) 169:6668-72.

35. Yamamoto $M$, Sato $S$, Hemmi $\mathrm{H}$, Hoshino K, Kaisho T, Sanjo $\mathrm{H}$, et al. Role of adaptor TRIF in the MyD88-independent tolllike receptor signaling pathway. Science (2003) 301:640-3. doi:10. 1126/science.1087262

36. Horng T, Barton GM, Flavell RA, Medzhitov R. The adaptor molecule TIRAP provides signalling specificity for toll-like receptors. Nature (2002) 420:329-33. doi:10. 1038/nature01180

37. Yamamoto $M$, Sato $S$, Hemmi $H$, Sanjo H, Uematsu S, Kaisho T, et al. Essential role for TIRAP in activation of the signalling cascade shared by TLR2 and TLR4. Nature (2002) 420:324-9. doi:10. 1038/nature01182

38. Aubry C, Corr SC, Wienerroither S, Goulard C, Jones R, Jamieson AM, et al. Both TLR2 and TRIF contribute to interferon-beta production during Listeria infection. PLoS One (2012) 7:e33299. doi:10.1371/ journal.pone.0033299

39. Poltorak A, Smirnova I, He X, Liu MY, Van Huffel C, McNally O, et al. Genetic and physical mapping of the Lps locus: identification of the toll- 4 receptor as a candidate gene in the critical region. Blood Cells Mol Dis (1998) 24:340-55. doi:10.1006/bcmd.1998.0201

40. Silhavy TJ, Kahne D, Walker S. The bacterial cell envelope. Cold Spring Harb Perspect Biol (2010) 2:a000414. doi:10.1101/ cshperspect.a000414

41. Raetz CR, Whitfield C. Lipopolysaccharide endotoxins. Annu Rev Biochem (2002) 71:635-700. doi:10.1146/annurev. biochem.71.110601.135414

42. Galanos C, Freudenberg MA. Mechanisms of endotoxin shock and endotoxin hypersensitivity. Immunobiology (1993) 187:346-56. doi:10.1016/S01712985(11)80349-9

43. Galanos C, Luderitz O, Rietschel ET, Westphal O, Brade $\mathrm{H}$, Brade $\mathrm{L}$, et al. Synthetic and natural Escherichia coli free lipid A express identical endotoxic activities. Eur J Biochem (1985) 148:1-5. doi:10. 1111/j.1432-1033.1985.tb08798.x

44. Matsuura M, Kawasaki K, Kawahara K, Mitsuyama M. Evasion of human innate immunity without antagonizing TLR4 by mutant Salmonella enterica serovar Typhimurium having penta-acylated lipid A. Innate Immun (2012) 18:764-73. doi:10.1177/1753425912440599

45. Lepper PM, Triantafilou M, Schumann C, Schneider EM, Triantafilou K. Lipopolysaccharides from Helicobacter pylori can act as 
antagonists for toll-like receptor 4. Cell Microbiol (2005) 7:519-28. doi:10.1111/j.1462-5822.2005. 00482.x

46. Ernst RK, Hajjar AM, Tsai JH, Moskowitz SM, Wilson CB, Miller SI. Pseudomonas aeruginosa lipid A diversity and its recognition by toll-like receptor 4 . J Endotoxin Res (2003) 9:395-400. doi: 10. 1177/09680519030090060201

47. Hajjar AM, Ernst RK, Tsai JH, Wilson CB, Miller SI. Human toll-like receptor 4 recognizes host-specific LPS modifications. Nat Immunol (2002) 3:354-9. doi:10.1038/ni777

48. Netea MG, van Deuren M, Kullberg BJ, Cavaillon JM, Van der Meer JW. Does the shape of lipid A determine the interaction of LPS with toll-like receptors? Trends Immunol (2002) 23:135-9. doi:10. 1016/S1471-4906(01)02169-X

49. Li Y, Powell DA, Shaffer SA, Rasko DA, Pelletier MR, Leszyk JD, et al. LPS remodeling is an evolved survival strategy for bacteria. Proc Natl Acad Sci U S A (2012) 109:8716-21. doi:10.1073/ pnas. 1202908109

50. Richards SM, Strandberg KL, Conroy $\mathrm{M}$, Gunn JS. Cationic antimicrobial peptides serve as activation signals for the Salmonella Typhimurium PhoPQ and PmrAB regulons in vitro and in vivo. Front Cell Infect Microbiol (2012) 2:102. doi:10.3389/fcimb.2012.00102

51. Prost LR, Miller SI. The Salmonellae PhoQ sensor: mechanisms of detection of phagosome signals. Cell Microbiol (2008) 10:576-82. doi:10.1111/j.1462-5822.2007. 01111.x

52. Farizano JV, Pescaretti Mde L, Lopez FE, Hsu FF, Delgado MA. The PmrAB systeminducing conditions control both lipid A remodeling and O-antigen length distribution, influencing the Salmonella Typhimurium-host interactions. J Biol Chem (2012) 287:38778-89. doi:10.1074/jbc.M112.397414

53. Kawasaki K, Ernst RK, Miller SI. Deacylation and palmitoylation of lipid A by Salmonellae outer membrane enzymes modulate host signaling through toll-like receptor 4 . I Endotoxin Res (2004) 10:439-44. doi:10.1179/096805104225006264

54. Ahrens B, Freund T, Rha RD, Dittrich AM, Quarcoo D, Hutloff A, et al. Lipopolysaccharide stimulation of dendritic cells induces interleukin-10 producing allergenspecific $\mathrm{T}$ cells in vitro but fails to prevent allergic airway disease.
Exp Lung Res (2009) 35:307-23. doi:10.1080/01902140802709460

55. Maaetoft-Udsen K, Vynne N, Heegaard PM, Gram L, Frokiaer H. Pseudoalteromonas strains are potent immunomodulators owing to low-stimulatory LPS. Innate Immun (2013) 19:160-73. doi:10. 1177/1753425912455208

56. Underhill DM, Ozinsky A, Hajjar AM, Stevens A, Wilson CB, Bassetti $\mathrm{M}$, et al. The toll-like receptor 2 is recruited to macrophage phagosomes and discriminates between pathogens. Nature (1999) 401:811-5. doi:10.1038/44605

57. Ying $\mathrm{H}, \mathrm{Da} L$, Yu-Xiu S, Yu X, Li-Xia L, Li-Mei X, et al. TLR4 mediates MAPK-STAT3 axis activation in bladder epithelial cells. Inflammation (2013) 36:1064-74. doi:10.1007/s10753-013-9638-7

58. Liu WH, Liu JJ, Wu J, Zhang LL, Liu F, Yin L, et al. Novel mechanism of inhibition of dendritic cells maturation by mesenchymal stem cells via interleukin-10 and the JAK1/STAT3 signaling pathway. PLoS One (2013) 8:e55487. doi:10. 1371/journal.pone.0055487

59. Meissner F, Scheltema RA, Mollenkopf HJ, Mann M. Direct proteomic quantification of the secretome of activated immune cells Science (2013) 340:475-8. doi:10. $1126 /$ science. 1232578

60. Sasai M, Yamamoto M. Pathogen recognition receptors: ligands and signaling pathways by toll-like receptors. Int Rev Immunol (2013) 32:116-33. doi:10.3109/08830185. 2013.774391

61. Aliprantis AO, Yang RB, Mark MR, Suggett S, Devaux B, Radolf $\mathrm{JD}$, et al. Cell activation and apoptosis by bacterial lipoproteins through toll-like receptor- 2 . Science (1999) 285:736-9. doi:10. 1126/science.285.5428.736

62. Morath S, Stadelmaier A, Geyer A, Schmidt RR, Hartung T. Synthetic lipoteichoic acid from Staphylococcus aureus is a potent stimulus of cytokine release. J Exp Med (2002) 195:1635-40. doi:10.1084/ jem.20020322

63. Peschel A, Otto M, Jack RW, Kalbacher H, Jung G, Gotz F. Inactivation of the dlt operon in Staphylococcus aureus confers sensitivity to defensins, protegrins, and other antimicrobial peptides. J Biol Chem (1999) 274:8405-10. doi:10.1074/jbc.274.13.8405

64. Zahringer $U$, Lindner $B$, Inamura S, Heine $\mathrm{H}$, Alexander C. TLR2 - promiscuous or specific? A critical re-evaluation of a receptor expressing apparent broad specificity. Immunobiology (2008) 213:205-24. doi:10.1016/j. imbio.2008.02.005

65. Hashimoto M, Furuyashiki M, Kaseya R, Fukada Y, Akimaru M, Aoyama K, et al. Evidence of immunostimulating lipoprotein existing in the natural lipoteichoic acid fraction. Infect Immun (2007) 75:1926-32. doi:10.1128/ IAI.02083-05

66. Jin MS, Kim SE, Heo JY, Lee ME Kim HM, Paik SG, et al. Crystal structure of the TLR1-TLR2 heterodimer induced by binding of a tri-acylated lipopeptide. Cell (2007) 130:1071-82. doi:10.1016/ j.cell.2007.09.008

67. Kang JY, Nan X, Jin MS, Youn SJ, Ryu YH, Mah S, et al. Recognition of lipopeptide patterns by tolllike receptor 2-toll-like receptor 6 heterodimer. Immunity (2009) 31:873-84. doi:10.1016/j.immuni. 2009.09.018

68. Boneca IG, Dussurget O, Cabanes D, Nahori MA, Sousa S, Lecuit $\mathrm{M}$, et al. A critical role for peptidoglycan N-deacetylation in Listeria evasion from the host innate immune system. Proc Natl Acad Sci U S A (2007) 104:997-1002. doi:10.1073/pnas.0609672104

69. Buwitt-Beckmann $U$, Heine $H$, Wiesmuller KH, Jung G, Brock R, Akira S, et al. Toll-like receptor 6-independent signaling by diacylated lipopeptides. Eur J Immunol (2005) 35:282-9. doi:10.1002/eji. 200424955

70. Kurokawa K, Kim MS, Ichikawa R Ryu KH, Dohmae N, Nakayama $\mathrm{H}$, et al. Environment-mediated accumulation of diacyl lipoproteins over their triacyl counterparts in Staphylococcus aureus. J Bacteriol (2012) 194:3299-306. doi:10. 1128/JB.00314-12

71. Frodermann V, Chau TA, Sayedyahossein S, Toth JM, Heinrichs DE, Madrenas J. A modulatory interleukin-10 response to staphylococcal peptidoglycan prevents Th1/Th17 adaptive immunity to Staphylococcus aureus. J Infect Dis (2011) 204:253-62. doi:10.1093/ infdis/jir276

72. Ozinsky A, Underhill DM, Fontenot JD, Hajjar AM, Smith $\mathrm{KD}$, Wilson $\mathrm{CB}$, et al. The repertoire for pattern recognition of pathogens by the innate immune system is defined by cooperation between toll-like receptors. Proc Natl Acad Sci U S A (2000) 97:13766-71. doi:10.1073/pnas.250476497
73. Takeuchi O, Kawai T, Muhlradt PF, Morr M, Radolf JD, Zychlinsky A, et al. Discrimination of bacterial lipoproteins by toll-like receptor 6. Int Immunol (2001) 13:933-40. doi:10.1093/intimm/13.7.933

74. Depaolo RW, Tang F, Kim I, Han $\mathrm{M}$, Levin $\mathrm{N}$, Ciletti $\mathrm{N}$, et al. Toll-like receptor 6 drives differentiation of tolerogenic dendritic cells and contributes to LcrVmediated plague pathogenesis. Cell Host Microbe (2008) 4:350-61. doi: 10.1016/j.chom.2008.09.004

75. Kurokawa K, Lee H, Roh KB, Asanuma M, Kim YS, Nakayama $\mathrm{H}$, et al. The triacylated ATP binding cluster transporter substratebinding lipoprotein of Staphylococcus aureus functions as a native ligand for toll-like receptor 2. J Biol Chem (2009) 284:8406-11. doi:10. 1074/jbc.M809618200

76. Chuang T, Ulevitch RJ. Identification of hTLR10: a novel human toll-like receptor preferentially expressed in immune cells. Biochim Biophys Acta (2001) 1518:157-61. doi:10.1016/S01674781(00)00289-X

77. Roach JC, Glusman G, Rowen L Kaur A, Purcell MK, Smith KD, et al. The evolution of vertebrate toll-like receptors. Proc Natl Acad Sci U S A (2005) 102:9577-82. doi:10.1073/pnas.0502272102

78. Guan Y, Ranoa DR, Jiang S, Mutha SK, Li X, Baudry J, et al. Human TLRs 10 and 1 share common mechanisms of innate immune sensing but not signaling. J Immunol (2010) 184:5094-103. doi:10.4049/jimmunol.0901888

79. Abad C, Gonzalez-Escribano MF, Diaz-Gallo LM, Lucena-Soto JM, Marquez JL, Leo E, et al. Association of toll-like receptor 10 and susceptibility to Crohn's disease independent of NOD2. Genes Immun (2011) 12:635-42. doi:10. 1038/gene.2011.41

80. Morgan AR, Lam WJ, Han DY, Fraser AG, Ferguson LR. Genetic variation within TLR10 is associated with Crohn's disease in a New Zealand population. Hum Immunol (2012) 73:416-20. doi: 10.1016/j.humimm.2012.01.015

81. Cario E. Barrier-protective function of intestinal epithelial tolllike receptor 2. Mucosal Immunol (2008) 1(Suppl 1):S62-6. doi:10. 1038/mi.2008.47

82. Durieux JJ, Vita N, Popescu O, Guette F, Calzada-Wack J, Munker $\mathrm{R}$, et al. The two soluble forms of the lipopolysaccharide receptor, CD14: characterization and release 
by normal human monocytes. Eur J Immunol (1994) 24:2006-12. doi: 10.1002/eji.1830240911

83. van Schravendijk MR, Handunnetti SM, Barnwell JW, Howard RJ. Normal human erythrocytes express CD36, an adhesion molecule of monocytes, platelets, and endothelial cells. Blood (1992) 80:2105-14.

84. Lee CC, Avalos AM, Ploegh HL. Accessory molecules for toll-like receptors and their function. Nat Rev Immunol (2012) 12:168-79. doi:10.1038/nri3151

85. Dziarski R, Tapping RI, Tobias PS. Binding of bacterial peptidoglycan to CD14. J Biol Chem (1998) 273:8680-90. doi:10.1074/jbc.273. 15.8680

86. Dziarski R, Gupta D. Staphylococcus aureus peptidoglycan is a toll-like receptor 2 activator: a reevaluation. Infect Immun (2005) 73:5212-6. doi:10.1128/IAI.73.8. 5212-5216.2005

87. Nakata T, Yasuda M, Fujita M, Kataoka H, Kiura K, Sano H, et al. CD14 directly binds to triacylated lipopeptides and facilitates recognition of the lipopeptides by the receptor complex of toll-like receptors 2 and 1 without binding to the complex. Cell Microbiol (2006) 8:1899-909. doi:10.1111/j. 1462-5822.2006.00756.x

88. Lee HK, Dunzendorfer S, Soldau K, Tobias PS. Double-stranded RNA-mediated TLR3 activation is enhanced by CD14. Immunity (2006) 24:153-63. doi:10.1016/j. immuni.2005.12.012

89. Baumann CL, Aspalter IM, Sharif O, Pichlmair A, Bluml S, Grebien $\mathrm{F}$, et al. CD14 is a coreceptor of toll-like receptors 7 and 9. J Exp Med (2010) 207:2689-701. doi:10. 1084/jem.20101111

90. Hoebe K, Georgel P, Rutschmann S, Du X, Mudd S, Crozat K, et al. CD36 is a sensor of diacylglycerides. Nature (2005) 433:523-7. doi:10.1038/nature03253

91. Dziarski R, Gupta D. Role of MD2 in TLR2- and TLR4-mediated recognition of gram-negative and gram-positive bacteria and activation of chemokine genes. J Endotoxin Res (2000) 6:401-5. doi:10. 1177/09680519000060050101

92. Dietrich N, Lienenklaus S, Weiss S, Gekara NO. Murine toll-like receptor 2 activation induces type I interferon responses from endolysosomal compartments.
PLoS One (2010) 5:e10250. doi:10. 1371/journal.pone.0010250

93. Santos-Sierra S, Deshmukh SD, Kalnitski J, Kuenzi P, Wymann MP, Golenbock DT, et al. Mal connects TLR2 to PI3Kinase activation and phagocyte polarization. EMBO J (2009) 28:2018-27. doi: 10.1038/emboj.2009.158

94. Troutman TD, Hu W, Fulenchek S, Yamazaki T, Kurosaki T, Bazan JF, et al. Role for B-cell adapter for PI3K (BCAP) as a signaling adapter linking toll-like receptors (TLRs) to serine/threonine kinases PI3K/Akt. Proc Natl Acad Sci U S A (2012) 109:273-8. doi:10.1073/ pnas.1118579109

95. Ni M, MacFarlane AW IV, Toft M, Lowell CA, Campbell KS, Hamerman JA. B-cell adaptor for PI3K (BCAP) negatively regulates tolllike receptor signaling through activation of PI3K. Proc Natl Acad Sci U S A (2012) 109:267-72. doi: 10.1073/pnas.1111957108

96. Triantis V, Trancikova DE, Looman MW, Hartgers FC, Janssen RA, Adema GJ. Identification and characterization of DC-SCRIPT, a novel dendritic cell-expressed member of the zinc finger family of transcriptional regulators. $J$ Immunol (2006) 176:1081-9.

97. Wang X, Dong L, Ni H, Zhou S, Xu Z, Hoellwarth JS, et al. Combined TLR7/8 and TLR9 ligands potentiate the activity of a Schistosoma japonicum DNA vaccine. PLoS Negl Trop Dis (2013) 7:e2164. doi:10.1371/journal.pntd.0002164

98. Hontelez S, Ansems M, Karthaus $\mathrm{N}$, Zuidscherwoude $\mathrm{M}$, Looman MW, Triantis V, et al. Dendritic cell-specific transcript: dendritic cell marker and regulator of TLRinduced cytokine production. $J$ Immunol (2012) 189:138-45. doi: 10.4049/jimmunol.1103709

99. Hofmann SR, Morbach H, Schwarz T, Rosen-Wolff A, Girschick HJ, Hedrich CM. Attenuated TLR4/MAPK signaling in monocytes from patients with CRMO results in impaired IL-10 expression. Clin Immunol (2012) 145:69-76. doi:10.1016/j.clim.2012.07.012

100. Ranoa DR, Kelley SL, Tapping RI. Human lipopolysaccharidebinding protein (LBP) and CD14 independently deliver triacylated lipoproteins to toll-like receptor 1 (TLR1) and TLR2 and enhance formation of the ternary signaling complex. J Biol Chem (2013) 288:9729-41. doi:10.1074/ jbc.M113.453266

101. Zaman M, Abdel-Aal AB, Phillipps KS, Fujita Y, Good MF, Toth I. Structure-activity relationship of lipopeptide group A streptococcus (GAS) vaccine candidates on toll-like receptor 2. Vaccine (2010) 28:2243-8. doi:10.1016/j.vaccine. 2009.12.046

102. Nguyen DT, Ludlow M, van Amerongen G, de Vries RD, Yuksel S, Verburgh RJ, et al. Evaluation of synthetic infection-enhancing lipopeptides as adjuvants for a live-attenuated canine distemper virus vaccine administered intranasally to ferrets. Vaccine (2012) 30:5073-80. doi:10.1016/j.vaccine. 2012.05.079

103. Nguyen DT, de Witte L, Ludlow M Yuksel S, Wiesmuller KH, Geijtenbeek TB, et al. The synthetic bacterial lipopeptide Pam3CSK4 modulates respiratory syncytial virus infection independent of TLR activation. PLoS Pathog (2010) 6:e1001049. doi:10.1371/journal. ppat.1001049

104. Tsai YG, Yang KD, Niu DM Chien JW, Lin CY. TLR2 agonists enhance CD8+Foxp3+ regulatory $\mathrm{T}$ cells and suppress Th2 immune responses during allergen immunotherapy. J Immunol (2010) 184:7229-37. doi:10.4049/ jimmunol.1000083

105. Chua BY, Pejoski D, Turner SJ, Zeng W, Jackson DC. Soluble proteins induce strong CD8+ $\mathrm{T}$ cell and antibody responses through electrostatic association with simple cationic or anionic lipopeptides that target TLR2. J Immunol (2011) 187:1692-701. doi:10.4049/jimmunol.1100486

106. Abdel-Aal AB, El-Naggar D, Zaman M, Batzloff $M$, Toth I. Design of fully synthetic, self-adjuvanting vaccine incorporating the tumor-associated carbohydrate $\mathrm{Tn}$ antigen and lipoamino acid-based tolllike receptor 2 ligand. $J$ Med Chem (2012) 55:6968-74. doi:10.1021/jm300822g

107. Long EM, Klimowicz AC, PaulaNeto HA, Millen B, McCafferty DM, Kubes P, et al. A subclass of acylated anti-inflammatory mediators usurp toll-like receptor 2 to inhibit neutrophil recruitment through peroxisome proliferatoractivated receptor gamma. Proc
Natl Acad Sci U S A (2011) 108:16357-62. doi:10.1073/pnas. 1100702108

108. Hooper LV. Bacterial contributions to mammalian gut development. Trends Microbiol (2004) 12:129-34. doi:10.1016/j.tim.2004.01.001

109. Mazmanian SK, Liu CH, Tzianabos AO, Kasper DL. An immunomodulatory molecule of symbiotic bacteria directs maturation of the host immune system. Cell (2005) 122:107-18. doi:10.1016/j. cell.2005.05.007

110. Mazmanian SK, Round JL, Kasper DL. A microbial symbiosis factor prevents intestinal inflammatory disease. Nature (2008) 453:620-5. doi:10.1038/nature07008

111. Atarashi K, Tanoue T, Oshima K, Suda W, Nagano Y, Nishikawa H, et al. Treg induction by a rationally selected mixture of clostridia strains from the human microbiota. Nature (2013) 500:232-6. doi:10.1038/nature12331

112. Nagano $\mathrm{Y}$, Itoh K, Honda K. The induction of Treg cells by gutindigenous clostridium. Curr Opin Immunol (2012) 24:392-7. doi:10. 1016/j.coi.2012.05.007

Conflict of Interest Statement: The authors declare that the research was conducted in the absence of any commercial or financial relationships that could be construed as a potential conflict of interest.

Received: 16 August 2013; accepted: 08 October 2013; published online: 28 October 2013.

Citation: Li J, Lee DSW and Madrenas J (2013) Evolving bacterial envelopes and plasticity of TLR2-dependent responses: basic research and translational opportunities. Front. Immunol. 4:347. doi: 10.3389/fimmu.2013.00347

This article was submitted to Microbial Immunology, a section of the journal Frontiers in Immunology.

Copyright $\odot 2013$ Li, Lee and Madrenas. This is an open-access article distributed under the terms of the Creative Commons Attribution License (CC BY). The use, distribution or reproduction in other forums is permitted, provided the original author(s) or licensor are credited and that the original publication in this journal is cited, in accordance with accepted academic practice. No use, distribution or reproduction is permitted which does not comply with these terms. 